\title{
Quantifying Conventional Electroencephalogram Recordings and Examining its Output Computation with a Quantitative Electroencephalogram
}

\author{
Gregory Xavier $^{1 *}$, Anselm Su Ting ${ }^{1} \&$ Norsiah Fauzan ${ }^{2}$ \\ ${ }^{1}$ Faculty of Medical and Health Sciences, Universiti Malaysia Sarawak, 94300 Kota Samarahan, \\ Sarawak, Malaysia \\ ${ }^{2}$ Faculty of Cognitive Sciences and Human Development, Universiti Malaysia Sarawak, 94300 \\ Kota Samarahan, Sarawak, Malaysia
}

\begin{abstract}
Quantitative electroencephalogram enables mathematical analysis of neurological recordings while conventional electroencephalogram lacks the mathematical output; hence, its usage is limited to neurological experts. This study was to determine if quantified conventional electroencephalogram recordings were compatible and comparable with quantitative electroencephalogram recordings. A group of post-call doctors was recruited and subjected to an EEG recording using a conventional electroencephalogram followed by a quantitative electroencephalogram device. The patterns and quantified recording results were compared. A comparative analysis of the two recording sets did not find differences in the recording patterns and statistical analysis. The findings promoted the use of a readily available conventional electroencephalogram in quantitative brain wave studies and have cleared potential compatibility bias towards data merging.
\end{abstract}

Keywords: quantitative electroencephalogram, conventional electroencephalogram, comparison, pattern, data merging

ARTICLE INFO

Email address: gregshc@yahoo.co.uk (Gregory Xavier)

*Corresponding author https://doi.org/10.33736/jcshd.3656.2021

e-ISSN: 2550-1623

Manuscript received: 16 July 2021; Accepted: 7 September 2021; Date of publication: 25 September 2021

Copyright: This is an open-access article distributed under the terms of the CC-BY-NC-SA (Creative Commons Attribution-NonCommercial-ShareAlike 4.0 International License), which permits unrestricted use, distribution, and reproduction in any medium, for non-commercial purposes, provided the original work of the author(s) is properly cited. 


\section{INTRODUCTION}

Conventional electroencephalogram (EEG) is widely used for clinical diagnosis of neurological disorders such as seizures, epilepsy, and unusual spells. It is an electrophysiological technique for the recording of electrical activity arising from the human brain. This device has temporal sensitivity, which evaluates the dynamic function of the brain (Louis et al., 2016).

Quantitative EEG (qEEG) recording refers to how EEG is digitised while mathematical values are extracted and used in many ways. qEEG is more sensitive than conventional EEG for detecting cerebral hemispheric abnormalities (Sürmeli, 2014:9).

Both types of EEG can detect abnormalities but cannot differentiate between kinds of pathology. EEG does not have localising ability as compared to MRI or CT scan. However, it can still be superficially suggestive. It is inexpensive, non-invasive, reproducible, and sensitive. It can be done in various settings and even continuously monitored (Nuwer \& Coutin-Churchman, 2012). Compared to conventional EEG devices, qEEG is also more user-friendly but may not be readily available. Moreover, it has not been clinically used in Malaysia.

Previous studies have indicated that qEEG has potential. It was found that qEEG analysis of neurological disorders and illness can improve diagnostic evaluations. However, up to the time of writing, no two previous studies had similar or developed a standardised method. It creates a difficult obstacle for the design and interpretation of obtained results collectively due to difficulties in comparing results across the literature (Jobert et al., 2012).

A few studies have notably compared and contrasted findings from conventional and qEEG results and found no significant differences. In addition, such studies had either quantified the conventional EEG recordings (Lodder \& van Putten, 2013) or compared them with other diagnostic neurological devices or machines (Schrooten et al., 2019). However, the methods used required specialised technical experts for analysis. The present study offers a much simpler method to assess the compatibility of the device for analysis.

The current study is part of a more extensive fatigue evaluation study by using EEG. In the larger evaluation fatigue study, cap qEEG was found to be a more user-friendly method. However, since it was not yet licensed to be used clinically in Malaysia, a comprehensive study was conducted using the conventional EEG to quantify its results. The reported findings hypothesised that there would not be any significant discrepancies between qEEG and quantified results from the conventional EEG. In addition, producing EEG devices would have gone through international manufacturing standards, ensuring that recordings obtained were valid.

\section{METHODOLOGY}

The study recruited 27 doctors from a tertiary general hospital who were all volunteers and had regular on-call duties. Their respective heads of departments were notified, and thus they were 
cleared to participate in the study. During the study, they were to conduct their duties as usual. The recordings were conducted after they had completed their on-call working hours. An on-call rotation officially begins at 8 am until the next day at $5 \mathrm{pm}$ overnight, equivalent to 33 working hours. The participants were screened, as they had to be identified to be free from any drug and alcohol influence or undergoing psychiatric treatment and management.

The participants were then required to attend two seatings of a $101 / 2$ minute EEG recording with three continuous sessions, including $31 / 2$ minutes of an open eye, close eye, reopen eye each (Hughes John 1999; Xavier et al. 2020). The recordings were carried out in a quiet room. Throughout the recordings, participants were seated while viewing a black dot on a computer screen. This study used two diverse types of EEG machines. Mitsar Cap quantitative EEG was used in the first seating, and a conventional Nicolet modal EEG by Natus was used in the second. The recordings were done on two different days due to logistics limitations and the participants' convenience and compliance.

These devices were the only EEG devices available for a more extensive study conducted on doctors' fatigue. The Nicolette EEG was registered under the Malaysian Medical Devices Act for clinical use, while Mitsar Cap-EEG was not. It was allowed only for research purposes.

The Mitsar Cap-EEG device was developed and made by Mitsar Co. Ltd., Russia. Its hardware and software are for clinical application and research use. However, it is yet to be used for clinical diagnostics in Malaysia and is limited only to research. Mitsar Co. Ltd. quality management system is certified to comply with the European standard for medical devices ISO 13485. Mitsar products have been classified as Class IIb devices and conform to the requirements of Annex I of the European Council Directive 93/42/EEC and are CE marked (Mitsar, 2019).

Mitsar Cap-EEG device was placed on the participant's head with its electrodes already in place, following the International 10-20 system using 19 channels. The brain electrical activity is measured in absolute power (microvolt, $\mu \mathrm{V} 2$ ) and hertz (frequency). The sampling rate was $1 \mathrm{kHz}$, with a low pass filter of $0.3 \mathrm{~Hz}$ and a high pass filter of $50 \mathrm{~Hz}$. The notch setting was at $45 \mathrm{~Hz}-55$ Hz. Electrical activity from the brain was recorded by using the average reference montage. The montage sums and averages the amplified outputs and uses them as a reference for each channel.

The model also comes with active noise cancellation. The technology reduces artefact waves associated with the movement of cables and environmental noise. The computation of spectral analysis is performed by a software called WinEEG, which the manufacturer provides. WinEEG software allows the recording, editing and analysis of continuously recorded EEG using a Mitsar amplifier (Biomedical, 2020). The software can be run by using the Microsoft Windows $10^{\mathrm{TM}}$ operating system. The process of EEG data acquisition and data analysis procedure is described in Figure 1 and Figure 2. 


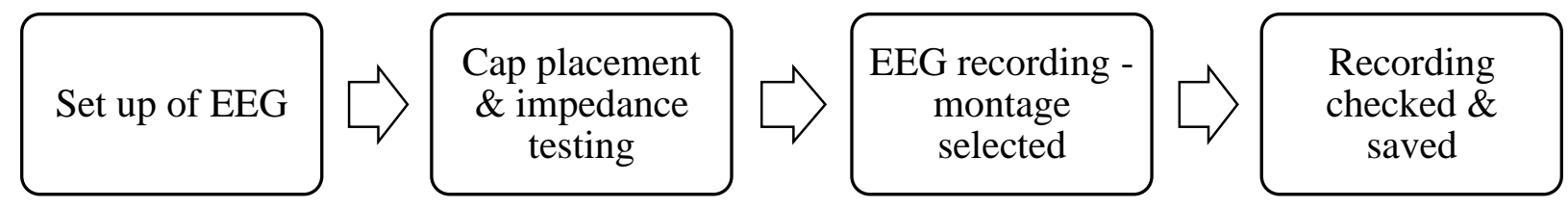

Figure 1. EEG Acquisition Steps (Mitsar)

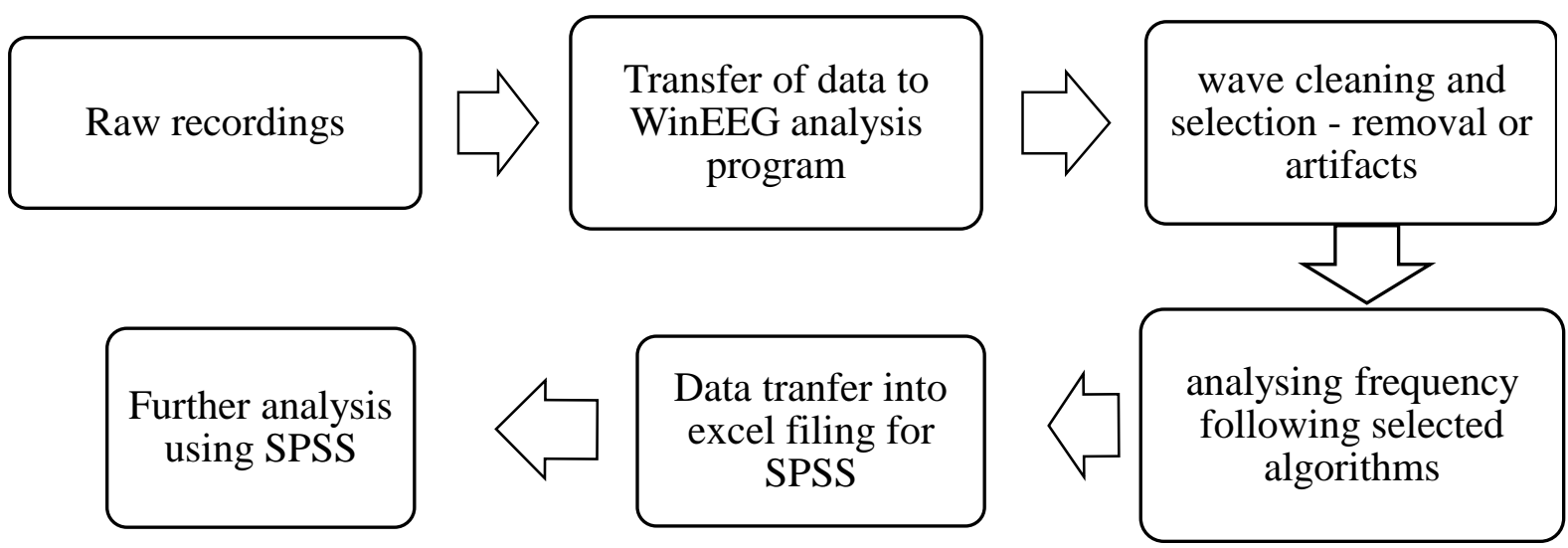

Figure 2. EEG Data Analysis Procedure (Mitsar)

The second EEG machine used is called Nicolette, and it was developed by Natus Neuro from the United States of America. It is not a cap-EEG. It is a conventional EEG machine and system, but clinicians commonly use it for diagnostic purposes. The electrodes were placed individually by using the International 10-20 system too. The Ten20 paste and Nuprep EEG skin prep gel were applied according to the manufacturer's instructions for use.

The brain electrical activity was measured in absolute power (microvolt, $\mu \mathrm{V} 2$ ) and hertz (frequency). The sampling rate was $1 \mathrm{kHz}$, with a low pass filter of $0.3 \mathrm{~Hz}$ and a high pass filter of $50 \mathrm{~Hz}$, and the notch setting was at $45-55 \mathrm{~Hz}$. The electrical activity from the brain was recorded by using the average reference montage. The montage sums and averages the amplified outputs and uses them to reference each channel (Juri D. Kropotov, 2009). These settings were like those used during the Mitsar cap-EEG recordings. The EEG acquisition and data analysis, as well as the conversion procedure, are as described in Figure 3 and Figure 4. The conventional EEG did not quantify the electrical activity. However, it was possible to export the data for conversion.

The raw Nicolette EEG data were exported in European Data Format (.edf) file format and converted into a (.eeg) file format using Mitsar EEG Studio Processing Software. It was after conversion that the recorded data were used by using the WinEEG software for further analysis. 


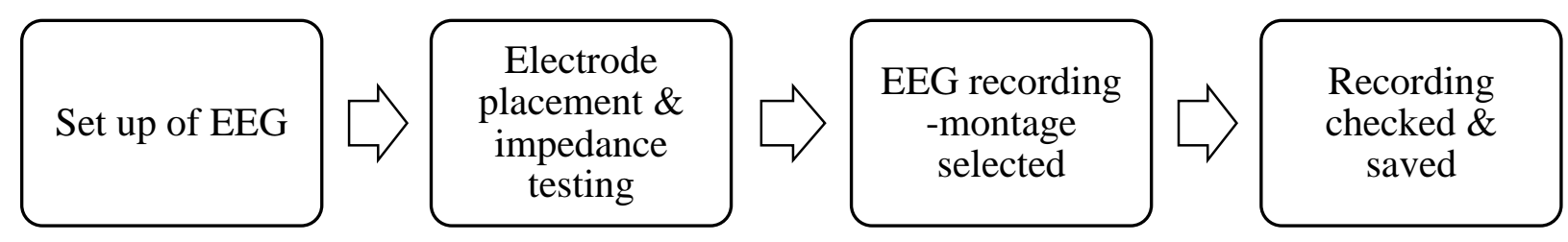

Figure 3. EEG Acquisition Steps (Nicolette)

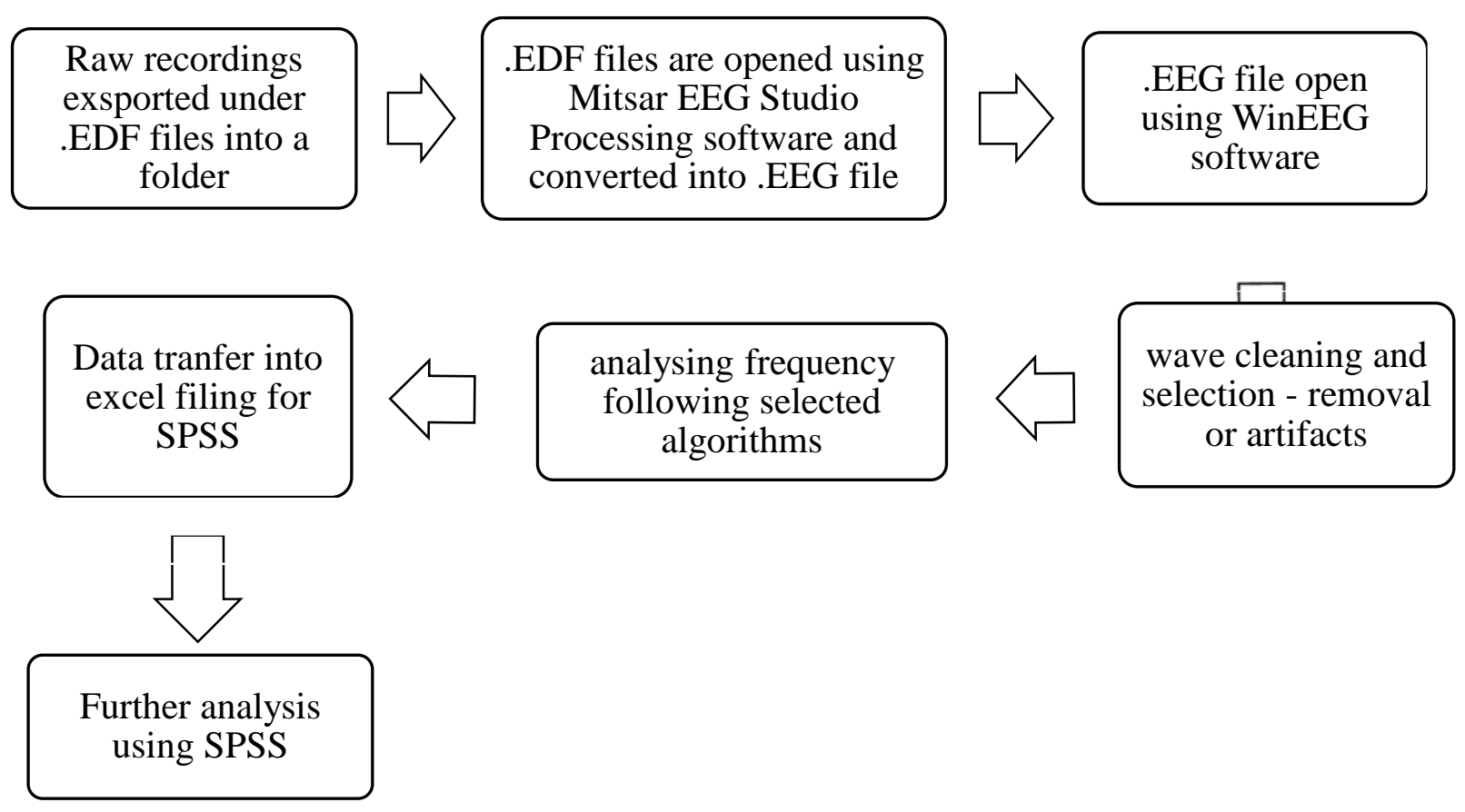

Figure 4. EEG Data Analysis Procedure (Nicolette)

\section{RESULTS}

A total of 27 participants underwent two EEG recordings using two different devices at the end of their post-call schedules on two different days. Participants consisted of 20 males and seven females with a mean age of 29.9 years. Their mean working experience duration is 4.8 years. The hours spent sleeping during their on-call period before the EEG recordings were not significantly different when paired t-test was used (Table 1). Since sleep is a major cofounding factor in brain wave changes, ensuring no significant differences would avoid bias in any recording result (Akerstedt, 1987; Akerstedt \& Wright, 2009; Xavier et al., 2020).

Figure 5 displays the pattern changes of brain wave electrical activity across the three different eye sessions. Figure 5 describes the brain waves electrical activity obtained from the two devices. The brain wave magnitudes of electrical activity obtained were the global average in microvolt square $\left(\mu \mathrm{V}^{2}\right)$. Table 2 is the numerical representation of the patterns observed in Figure 5 and the p-value. 
In general, those observed patterns showed similarity in changes with no significant differences between quantified values from the conducted paired t-test.

Theta increase from opened eye to closed eye with Mitsar was slightly higher than Nicolet, but the differences were not significant. From closed to the reopened eye, the decrease was more or less the same and thus was not significant. The pattern of change was observed amongst those who were deprived of sleep compared to those who were not customarily observed to have a decrease in magnitude from opened eye to closed eye (Forest \& Godbout, 2000; Strijkstra et al., 2003).

Alpha was increased from opened eye to closed eye and then decreased to the reopened eye. The phenomenon occurred both in the Mitsar and Nicolet recordings. However, the decrease from closed to reopened was slightly higher with Nicolet, but the difference was not significant. Beta electrical activity from Nicolet recordings had a progressive increase, while Mitsar recordings had a slight dip in electrical activity during close eye that increased later during reopen eye session. The decrease in electrical activity from closed eye to reopened eye for the alpha wave was higher in Nicolet recordings than from Mitsar recordings. These alpha and beta patterns matched the normalcy of brain wave changes at rest (Juri D. Kropotov, 2009).

The algorithms showed no actual difference during the different sessions and while transitioning. Algorithmic calculation averages and demonstrates the overall differences of any two groups of factors. Any difference between the Nicolet and Mitsar recordings could be elicited by using the calculation, but such was not observed. However, relative theta and relative alpha recordings only had a significant difference with a closed eye $(p<0.001)$ (Table 2$)$.

Table 1. Demographic distribution of participants $(n=27)$

\begin{tabular}{lc}
\hline Age (yrs) & 29.9 \\
Mean & 30.0 \\
Median & 1.8 \\
SD & 27.0 \\
Min & 35.0 \\
Max & \\
Sex & 20 \\
Male & 7 \\
Female & \\
Work duration (yrs) & \\
Mean & 4.8 \\
Median & 4.5 \\
SD & 2 \\
Min & 2.0 \\
Max & 10.0
\end{tabular}


Journal of Cognitive Sciences and Human Development. Vol.7(2), September 2021

\begin{tabular}{lcc} 
Sleep duration $(\mathrm{hrs})$ & Mitsar & Nicolet \\
Mean & 1.9 & 2.1 \\
Median & 2.0 & 2.0 \\
SD & 0.96 & 0.87 \\
Min & 0.5 & 1.0 \\
Max & 4.0 & 4.0 \\
\hline
\end{tabular}




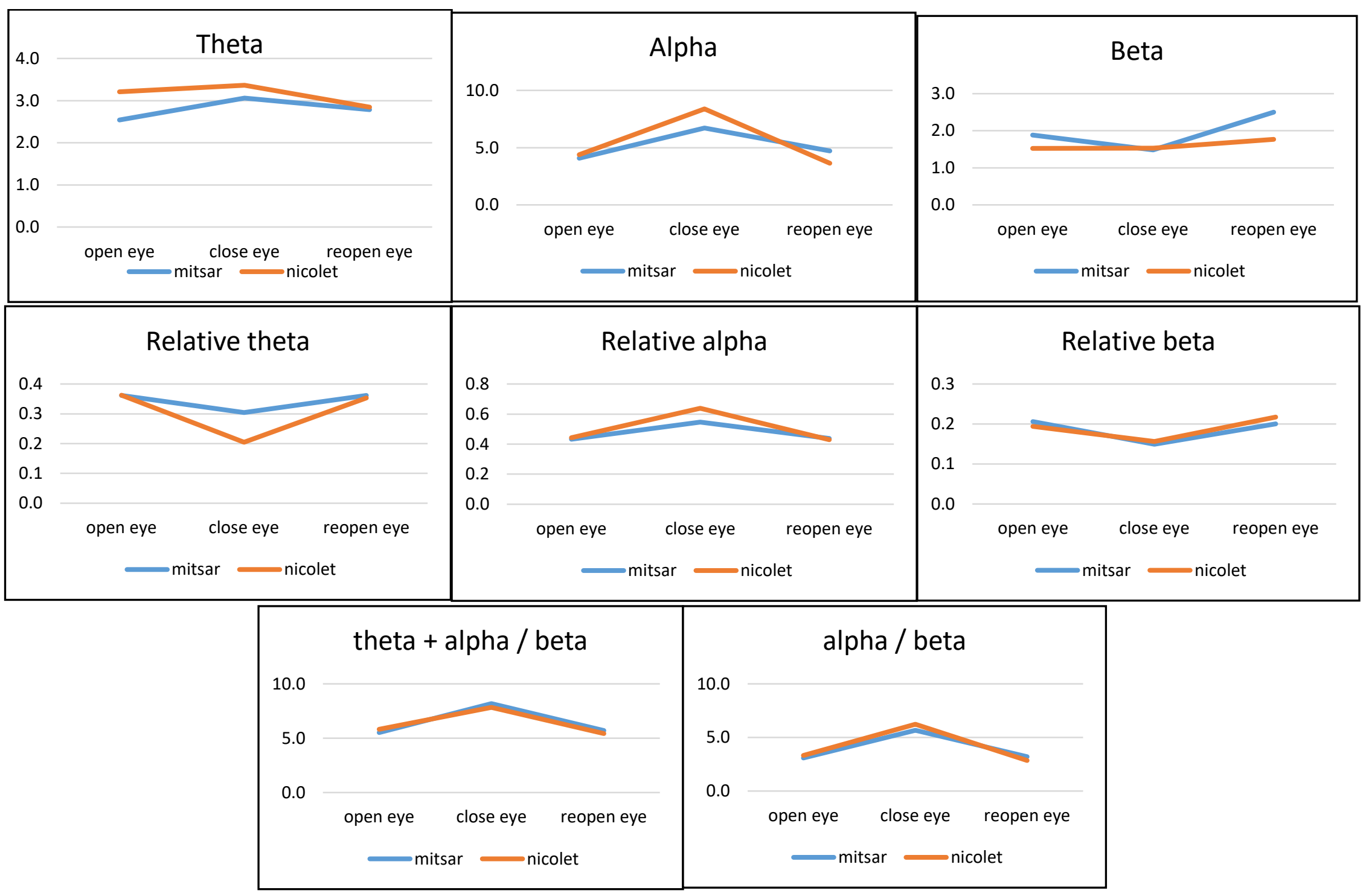

Figure 5. Patterns of brain wave electrical activity from Mitsar and Nicolet EEG recordings 
Table 2. Global brain wave electrical activity recordings of Mitsar and Nicolet EEG devices with (microvolts $-\mu \mathrm{V}^{2}$ )

\begin{tabular}{|c|c|c|c|c|}
\hline & & Mitsar & Nicolet & p-value \\
\hline \multirow{3}{*}{ Theta } & Open eye & 2.540 & 3.210 & 0.280 \\
\hline & close & 3.062 & 3.365 & 0.811 \\
\hline & reopen & 2.787 & 2.844 & 0.929 \\
\hline \multirow{3}{*}{ Alpha } & Open eye & 4.077 & 4.380 & 0.836 \\
\hline & close & 6.714 & 8.382 & 0.477 \\
\hline & reopen & 4.730 & 3.638 & 0.522 \\
\hline \multirow{3}{*}{ beta } & Open eye & 1.881 & 1.524 & 0.606 \\
\hline & close & 1.482 & 1.530 & 0.903 \\
\hline & reopen & 2.500 & 1.768 & 0.533 \\
\hline \multirow{3}{*}{ Relative theta } & Open eye & 0.362 & 0.363 & 0.974 \\
\hline & close & 0.304 & 0.204 & $0.000^{*}$ \\
\hline & reopen & 0.362 & 0.353 & 0.787 \\
\hline \multirow{3}{*}{$\begin{array}{l}\text { Relative } \\
\text { alpha }\end{array}$} & Open eye & 0.432 & 0.443 & 0.775 \\
\hline & close & 0.546 & 0.639 & $0.007 *$ \\
\hline & reopen & 0.438 & 0.429 & 0.819 \\
\hline \multirow{3}{*}{ Relative beta } & Open eye & 0.206 & 0.194 & 0.627 \\
\hline & close & 0.150 & 0.156 & 0.630 \\
\hline & reopen & 0.200 & 0.217 & 0.464 \\
\hline \multirow{3}{*}{$\begin{array}{l}\text { Alpha + theta } \\
/ \text { beta }\end{array}$} & Open eye & 5.517 & 5.817 & 0.776 \\
\hline & close & 8.182 & 7.828 & 0.708 \\
\hline & reopen & 5.715 & 5.419 & 0.760 \\
\hline \multirow{3}{*}{ Alpha/beta } & Open eye & 3.078 & 3.306 & 0.732 \\
\hline & close & 5.672 & 6.227 & 0.547 \\
\hline & reopen & 3.194 & 2.842 & 0.466 \\
\hline
\end{tabular}

$*$ p-value $<0.001$ with paired t-test

\section{DISCUSSION}

The recordings conducted in this study were deliberately carried out after the participants had completed their on-call duties. Therefore, they were usually sleep-deprived, which was exhibited in the study. It was reported before that those who were sleep-deprived exhibited brain wave changes detected by EEG. Sleep deprivation had affected the magnitude of brain wave electrical activity and minimally over its pattern of transition between sessions (Ferreira et al., 2006; Klimesch, 1999). Therefore, if the two EEG devices were compatible, such changes should also be evident. 
The EEG changes seen across sessions were normal occurrences, except for theta changes influenced by sleep deprivation. During closed eye, it is typical for the alpha electrical activity to increase while the beta decreases. The alpha electrical activity decreased after the reopened eye session while the beta increased again (Juri D. Kropotov, 2009). As for theta, usually, it changes from opened eye to the closed eye should be in opposite alpha changes occurrence. However, both devices managed to capture the increase in theta magnitude in contrast to a decrease.

The algorithms adopted in this study was created from a previous study on fatigue to detect changes between transitions and in different sessions, as adopted in the present study (Lal et al., 2003). Using such algorithms, including measuring the relative differences, exhibited any actual differences (Lal \& Craig, 2002; shyh-Yueh Cheng, 2007). This study used the same principle and found no differences in pattern and brain electrical activity between the two recorded devices results.

This study was conducted on two different days, opening to bias and the effects of confounding factors. Nevertheless, there was no significant difference in the targeted confounding factor of sleep. Furthermore, the participants were recruited while on active duty and due to the need to maintain their compliance to the study, two days were needed. This prospects another study to be conducted in controlled environments and recordings done on the same day. To further enhance the agreement of quantified conventional EEG recording and quantitative EEG devices, such a study should be carried out using other methods and other groups of people.

In general, there were no significant differences between the device recordings conducted on different days, except for relative theta and relative alpha closed eye sessions. Such possibilities could arise due to inter-individual differences or distant aberrations in recordings (Klimesch, 1999). Klimesch (1999) also argued that the inter-individual difference could be individuals with different baselines brain wave electrical activity. In addition, it could be factored by age, experience, mood, intelligence, experience or even physiological to which this study could not find those effects (Grandy et al., 2013). To adjust these differences, algorithms were formulated by Sauvet et al. (2014) and Picot et al. (2008). in this study, the alpha + theta/beta and alpha/beta algorithm results were almost identical between devices. However, this study did not further analyse the brain waves by using spectral analysis.

Previous studies have not attempted to conduct a method such as in this study. Instead, reviewing routine EEGs were carried out based usually on visual analysis of the raw signals (Lodder \& van Putten, 2013). Then the studies assessed and evaluated the consistencies in reporting between conventional EEG and qEEG (spectral analysis) results. These required specialised experts. Nevertheless, those studies concluded that the outcomes were reliable, revolving around psychiatric studies, but was aborted as it still required proper guidelines to reduce bias (Hughes \& John, 1999; Lodder \& van Putten 2013). Hughes and John (1999) stated that qEEG studies were particularly well suited to identify subtle changes in the topographic distribution of background activity and aid complex differential diagnoses. 
The study concludes that the conventional EEG device (Nicolet) can be quantified and be used in tandem with quantitative EEG (Mitsar). Both devices have been found to be able to manage the detection of brain wave changes that occur during sleep deprivation. Such an agreement promotes the use of a much readily available conventional EEG in quantitative brain wave studies. In addition, it clears the compatibility bias of data merging between devices. The devices quantified data can be merged. The proper software could also be carried out a spectral and topographic analysis of quantified conventional EEG data. With the advancement of technology, EEG usage can now be carried out by various experts of concern on clinical patients.

\section{ACKNOWLEDGEMENTS AND DISCLOSURE}

The study is registered with the National Medical Research Registry, and ethical approval was obtained from its Medical Research Ethics Committee (MREC). Informed consent was obtained from all respondents. The Malaysian Brain, Mind and Neuroscience Research Foundation has provided a grant for this study. The authors declared no conflict of interest in conducting the study regarding any companies implicated in this research study. We acknowledge the Director-General of Health Malaysia for allowing the Ministry of Health facilities and staff (Sarawak General Hospital Neurology Unit and Clinical Research Centre). The authors thank Universiti Malaysia Sarawak for its support and use of its resources.

\section{REFERENCES}

Akerstedt, T., \& Kenneth P. W. (2009). Sleep Loss and Fatigue in Shift Work and Shift Work Disorder. Sleep Medicine Clinics, 4(2), 257-271. doi:10.1016/j.jsmc.2009.03.001

Akerstedt, T. (1987). Sleep/Wake Disturbances in Working Life. Electroencephalography and Clinical Neurophysiology, 39, 360-363.

Aminoff, M.J. (2012) London. In Nuwer, Marc R., and Pedro Coutin-Churchman (Ed.), Chapter 8 - Topographic Mapping, Frequency Analysis, and Other Quantitative Techniques in Electroencephalography. (pp. 187-206) in Aminoff's Electrodiagnosis in Clinical Neurology (Sixth Edition), London: W.B. Saunders. ISBN: 9781455726769

Antoine, P., Charbonnier, S., \& Caplier, A. (2008). On-Line Automatic Detection of Driver Drowsiness Using a Single Electroencephalographic Channel. Annual International Conference of the IEEE Engineering in Medicine and Biology Society. IEEE Engineering in Medicine and Biology Society. Annual International Conference, 3864-3867. doi:10.1109/IEMBS.2008.4650053

Bio-medical. (2020, October 14). WinEEG Advanced Software for Mitsar. Bio-Medical-Mitsar Retrieved from https://bio-medical.com/wineeg-advanced-software-for-mitsar.html.

Ferreira, C., Deslandes, A. Moraes, H., \& Cagy, M. (2006). Electroencephalographic Changes after One Night of Sleep Deprivation. Arquivos de Neuro-Psiquiatria, 64(2B), 388-393. doi:10.1590/S0004-282X2006000300007 
Forest, G., \& Godbout, R. (2000). Effects of Sleep Deprivation on Performance and EEG Spectral Analysis in Young Adults. Brain and Cognition, 43(1-3), 195-200.

Grandy, T. H., Werkle-Bergner, M., Chicherio, C., Schmiedek, F., Lövdén, M., \& Lindenberger, U. (2013). Peak Individual Alpha Frequency Qualifies as a Stable Neurophysiological Trait Marker in Healthy Younger and Older Adults. Psychophysiology, 50 (6), 570-582. doi:10.1111/psyp.12043

Hughes, J. R., \& E. R. John. (1999). Conventional and Quantitative Electroencephalography in Psychiatry. The Journal of Neuropsychiatry and Clinical Neurosciences, 11(2),190-208. doi:10.1176/jnp.11.2.190

Jobert, M., Frederick J. W., Ruigt, Gé S. F., Brunovsky, M., Prichep, L.S., \& Wilhelmus H. I. M. (2012). Guidelines for the Recording and Evaluation of Pharmaco-EEG Data in Man: The International Pharmaco-EEG Society (IPEG). Neuropsychobiology, 66(4), 201-220. doi:10.1159/000343478

Kropotov, J.D. (2009). Quantitative EEG, Event-Related Potentials and Neurotherapy (1st ed. Academic Press) United States: New York, Elsevier.

Klimesch, W. (1999). EEG Alpha and Theta Oscillations Reflect Cognitive and Memory Performance: A Review and Analysis. Brain Research. Brain Research Reviews, 29(2-3), 169195. doi:10.1016/s0165-0173(98)00056-3

Lodder, S. S., Michel J. A. M., \& Van-Putten (2013). Quantification of the Adult EEG Background Pattern. Clinical Neurophysiology: Official Journal of the International Federation of Clinical Neurophysiology, 124(2), 228-237. doi:10.1016/j.clinph.2012.07.007

Louis, E. K., Lauren, S. C. F., Jeffrey, W. B., Jennifer L. H., Korb, P., Mohamad Z., Koubeissi, W. E., Lievens, E. M., Knight, P., \& St Louis, E.K. (2016). Electroencephalography (EEG): An Introductory Text and Atlas of Normal and Abnormal Findings in Adults, Children, and Infants. $\left(1^{\text {st }}\right.$ ed. American Epilepsy Society) Chicago, IL: American Epilepsy Society. https://dx.doi.org/10.5698/978-0-9979756-0-4

Mitsar. (2019, October 14). About Us - Mitsar: Neurodiagnostics : Electroencephalography (EEG). Mitsar, Brain Diagnostics Solutions. Retrieved from https://mitsar-eeg.com/about-us/.

Saroj K. L., \& Craig, A. (2002). Driver Fatigue: Electroencephalography and Psychological Assessment. Psychophysiology, 39(3), 313-321. doi:10.1017/S0048577201393095

Saroj K. L., Craig, A., Boord, P., Kirkup, L., \& Nguyen, H. (2003). Development of an Algorithm for an EEG-Based Driver Fatigue Countermeasure. Journal of Safety Research, 34(3), 321-328. doi:10.1016/S0022-4375(03)00027-6

Sauvet, F., Bougard, C., Coroenne, M., Lely, L., Van-Beers, P., Elbaz, M., Guillard, M., Leger, D., \& Chennaoui, M. (2014). In-Flight Automatic Detection of Vigilance States Using a Single 
EEG Channel. IEEE Transactions on Bio-Medical Engineering, 61(12), 2840-47. doi:10.1109/TBME.2014.2331189

Shyh-Yueh Cheng. (2007). Electroencephalographic Study of Mental Fatigue in Visual Display Terminal Task. Journal of Medical and Biological Engineering, 27(3), 124-131.

Strijkstra, A. M., Domien G. M. Beersma, B. D., Halbesma, N., \& Daan, S. (2003). Subjective Sleepiness Correlates Negatively with Global Alpha (8-12 Hz) and Positively with Central Frontal Theta (4-8 Hz) Frequencies in the Human Resting Awake Electroencephalogram. Neuroscience Letters, 340 (1), 17-20. doi:10.1016/s0304-3940(03)00033-8

Sürmeli, T. (2014). Chapter Nine - Treating Thought Disorders. (pp. 213-51). Clinical Neurotherapy, edited by D. S. Cantor and J. R. Evans. Boston: Academic Press. doi.org/10.1016/B978-0-12-396988-0.00009-X

Vandenberghe, M.R., Peeters R., \& Dupont, P. (2019). Quantitative Analyses Help in Choosing Between Simultaneous vs. Separate EEG and FMRI. Frontiers in Neuroscience, 12: 1009. doi:10.3389/fnins.2018.01009

Xavier, G., Anselm, S. T., \& Fauzan, N. (2020). Exploratory Study of Brain Waves and Corresponding Brain Regions of Fatigue On-Call Doctors Using Quantitative Electroencephalogram. Journal of Occupational Health, 62(1), 1-8. doi:10.1002/13489585.12121 